\title{
Morphogenesis and dynamics of slime molds in various environments
}

5

6

$7 \quad$ Fernando Patino-Ramirez ${ }^{1^{*}}$, Aurèle Boussard ${ }^{2}$, Chloé Arson $^{1 \pi}$, Audrey Dussutour ${ }^{2 \pi^{*}}$

8

9

10

11

12

1 School of Civil and Environmental Engineering, Georgia Institute of Technology, Atlanta, GA, USA

13

14

2

15

University, CNRS, UPS, Toulouse, France

16

17

18

19

20

$21{ }^{*}$ Corresponding authors

22 E-mails: fp@gatech.edu (FP), audrey.dussutour@univ-tlse3.fr (AD)

23

24

$25 \pi$ These authors contributed equally to this work.

26

27 


\section{Abstract}

30 Cells, including unicellulars, are highly sensitive to external constraints from their

31 environment. Amoeboid cells change their cell shape during locomotion and in 32 response to external stimuli. Physarum polycephalum is a large multinucleated 33 amoeboid cell that extends and develops pseudopods. In this paper, changes in cell

34 behavior and shape were measured during the exploration of homogenous and non35 homogenous environments that presented neutral, and nutritive and/or adverse 36 substances. In the first place, we developed a fully automated image analysis method to measure quantitatively changes in both migration and shape. Then we measured various metrics that describe the area covered, the exploration dynamics, the migration rate and the slime mold shape. Our results show that: 1) Not only the nature, but also the spatial distribution of chemical substances affect the exploration behavior of slime molds; 2) Nutritive and adverse substances both slow down the exploration and prevent the formation of pseudopods; and 3) Slime mold placed in an adverse environment preferentially occupies previously explored areas rather than unexplored areas using mucus secretion as a buffer. Our results also show that slime molds migrate at a rate governed by the substrate up until they get within a critical distance to chemical substances.

Key words: Morphogenesis, Slime molds, Nutritive/adverse environments, Image analysis, Migration rate, Growth, Exploration 


\section{Author summary}

55 Physarum polycephalum, also called slime mold, is a giant single-celled organism 56 that can grow to cover several square meters, forming search fronts that are 57 connected to a system of intersecting veins. An original experimental protocol 58 allowed tracking the shape of slime mold placed in homogenous substrates 59 containing an attractant (glucose) or a repellent (salt), or inhomogeneous substrates 60 that contained an attractive spot (glucose), an eccentric slime mold and a repulsive spot (salt) in between. For the first time, the rate of exploration of unexplored areas (primary growth) and the rate of extension in previously explored areas (secondary growth) were rigorously measured, by means of a sophisticated image analysis program. This paper shows that the chemical composition of the substrate has more influence on the morphology and growth dynamics of slime mold than that of concentrated spots of chemicals. It was also found that on a repulsive substrate, slime mold exhibits a bias towards secondary growth, which suggests that the mucus produced during slime mold migration acts as a protective shell in adverse environments.

\section{Introduction}

73 Large-scale spatial patterns in biology are common and knowing how these patterns

74 evolve and what are their functional role, enables us to understand the evolution of 75 biocomplexity (see e.g. (1-4)). Morphogenesis has been studied in length at the cell 76 level (see e.g (5-8)); cells are highly sensitive to geometrical and mechanical 77 constraints from their microenvironment and respond to these conditions by 
78 changing shape (see e.g. $(9,10)$ ); these transformations impact cell migration and

79 growth (see e.g. (7,11-13) ). Cellular migration is a fundamental property of every cell and it is crucial for the development and morphogenesis of animal body plans and organ systems (see e.g. (14-16) ). Cell migration is either in a random direction or directed towards localized cues (17-20). Mechanisms of cellular movement have been mostly studied in chemotactic cells, such as neutrophils (17), bacteria (21), Ciliata (22), fungi (23) and cellular slime molds (19).

Due to its extremely fast migration rate and highly irregular shape, the acellular slime mold Physarum Polycephalum represents a prime example of differentiated growth and thus offers an attractive model for the analysis of morphogenesis dynamics underlying cellular migration and exploration (24-28). $P$ polycephalum is a giant single-celled organism that can grow to cover several square meters. Its morphology includes search fronts that are connected to a system of intersecting veins, in which oscillatory flows of the protoplasm "shuttle streaming" take place. This vein network allows 1) an efficient distribution of chemical signals, oxygen, nutrients over large distances and 2) cell migration at a speed of few centimeters per hour $(29,30)$. The driving force for this protoplasm streaming is a periodic, peristaltic contraction and relaxation of the veins due to the actin-myosin interaction, which is regulated by

97 oscillations of intracellular chemicals such as calcium (31-33). As it explores its 98 environment, the slime mold extends temporary arm-like projections named pseudopods. It also secretes continuously a thick extracellular slime (34). The glycoprotein nature of the extracellular slime coat endows $P$ polycephalum with 101 unique protective and structural properties that favor survival of the migrating, naked 
102 slime mold (35). As the slime mold is foraging, it avoids areas covered with this

103 mucus, which marks previously explored areas $(36,37)$.

104

105 In the presence of chemical substances in the environment, $P$. Polycephalum shows

106 directional movements towards or away from the stimuli (i.e. chemotaxis). Physarum

107 morphology, evolution and behaviors are strongly affected by the availability, location

108 and concentration of nutrients. When the slime mold senses attractants (e.g. food

109 cues) using specific receptors located on the membrane, the oscillation frequency in

110 the pseudopod closest to the attractant increases, causing cytoplasm to flow towards

111 the attractant (38). On the contrary, when repellents such as salts are sensed, the

112 oscillation frequency decreases and the slime mold moves away from the repellent

113 (38). Although slime molds lack the complex hardware of animals with brains, they

114 live in environments that are as complex and they face the same decision-making

115 challenges (39). Hence, acellular slime molds have been the subject of a wide range

116 of studies showing that they can solve complex biological and computational

117 problems without any specialized nervous tissue $(24,36,37,40-45)$.

118

119 In this paper the objectives are to characterize the morphology and dynamics of

120 Physarum exploring various environments. First, we investigate how movement is

121 affected by homogeneous environmental conditions: adverse environment (using salt

122 as a repellent (46); nutritive environments (using glucose as a chemo-attractant

$123(47,48)$ with 2 different concentrations) and a neutral environment (using plain agar).

124 Second, we analyze the geometrical evolution of slime molds placed at a distance

125 from a nutritive spot (glucose), with and without a repelling spot (salt) in between.

126 We characterize slime molds' movement both temporally and spatially, to capture the 
127 full dynamics. To this aim, we develop a program that automatically analyzes

128 sequences of images to track the areas covered and explored by the slime mold, the

129 slime mold shape, the refinement and secondary growth cycles, as well as the

130 distance to the nutritive spot.

131

\section{Results}

\section{1) Homogeneous environment}

134 In order to study the influence of the environment on slime mold expansion rate, we

135 analyzed the areas covered by slime mold, unexplored substrate and mucus over

136 time, as shown in Fig 1.
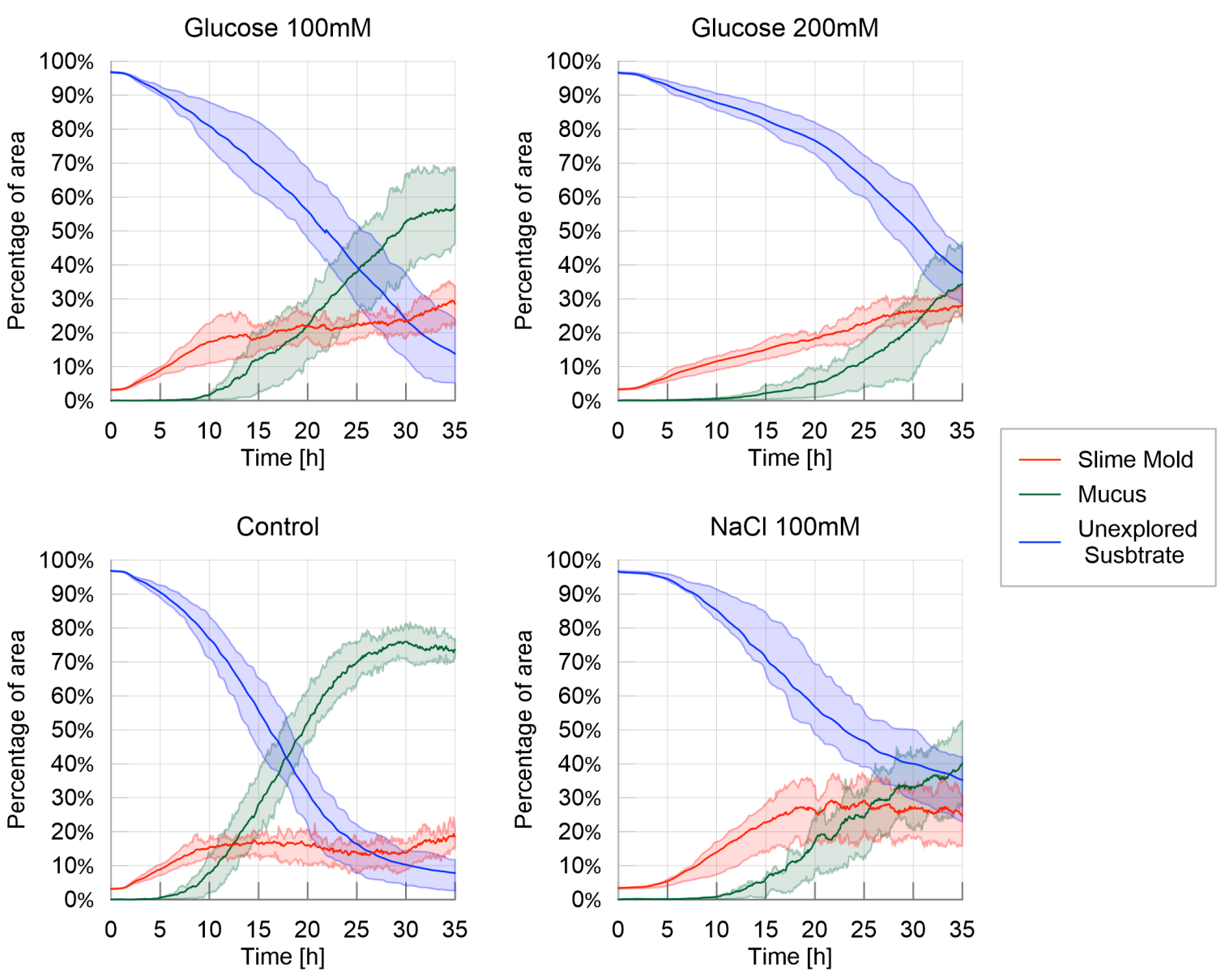

137 
138 Fig 1. Fraction of area covered by slime mold, mucus and unexplored substrate - Homogeneous

139 environment. The solid line corresponds to the average index calculated over the 20 replicates, while

140 the shaded areas correspond to the first and third quartiles of the data.

141

142 In a neutral (control) and slightly nutritive environment (glucose at $100 \mathrm{mM}$ ), the

143 slime molds started to spread from the very beginning of the experiment (Fig 1;

144 Table 1 and Fig 5 in S1 Appendix, $P>0.05)$. In contrast in a highly nutritive

145 environment (200 mM glucose), slime molds started to explore later $(P<0.001$ when

146 compared to the control). Slime molds placed in an adverse environment (100 mM

$147 \mathrm{NaCl})$ were lagging the most and only started exploring after 3 hours $(P<0.001$ when

148 compared to the control). Once the slime molds started to explore, they all grew at

149 the same rate (Fig 1; Table 2, Fig 6 in S1 Appendix, P> 0.05 when compared to the

150 control) except the ones placed in a highly nutritive environment which were slowed

151 down $(P<0.001$ when compared to the control).

152 At the end of the experiment (after 35 hours), the slime molds reached a similar

153 surface in a control environment and in an adverse environment (Table 3 and Fig 7

154 in S1 Appendix , P>0.05 when compared to the control). Interestingly, after reaching

155 a plateau at 18 hours, the area covered by the slime molds in an adverse

156 environment oscillated with seemingly cyclic fluctuations (Fig 1). In both a slightly

157 and a highly nutritive environment, the slime molds reached a higher final surface

158 than the slime molds placed in a control environment $(P<0.001$ in both comparisons)

159 and covered approximately $30 \%$ of arena at the end experiment. It is worth noting

160 that in a highly nutritive environment, the surface covered by the slime molds never

161 reached a plateau after 35 hours, suggesting that the slime molds did not reach its

162 maximum surface (Fig 1). 
164 Refinement i.e. appearance of mucus, was observed after 5 hours in the control environment. In all other environments, mucus appeared later (Table 4 and Fig 8 in

166 S1 Appendix: $\mathrm{P}<0.001$ for all treatments when compared to the control). In a highly 167 nutritive environment, mucus was only observed after 10 hours, which marked the 168 strongest delay in the refinement process. Once the mucus started to be apparent, 169 its surface grew quicker in the control environment than in the other three treatments 170 (Table 5 and Fig 9 in S1 Appendix; $\mathrm{P}<0.001$ for all treatments when compared to the 171 control). Thus the surface covered by mucus at the end of the experiment was the 172 largest in the control environment where it reached $75 \%$ of the arena against $55 \%$, $17340 \%$ and $35 \%$ for the slightly nutritive, the adverse and the highly nutritive 174 environments respectively (Table 6 and Fig 10 in S1 Appendix; $P<0.001$ for all 175 treatments when compared to the control).

177 Hence, slime molds placed in a control environment explored almost all the arena 178 leaving only $5 \%$ of the arena unexplored while in the other treatments the surface 179 unexplored were significant: $15 \%, 35 \%$ and $38 \%$ for the slightly nutritive, the highly nutritive and the adverse environments respectively. Interestingly, although the

181 growth rate dynamics differed between highly nutritive and adverse environments, 182 the final unexplored surfaces were similar. In a highly nutritive environment the slime 183 molds grew slowly and steadily while in an adverse environment slime molds grew 184 rather quickly but after a long delay.

186 Next, we analyzed the evolution of the cumulative areas covered by primary growth, 187 refinement and secondary growth (Fig 2). The cumulative area covered by 188 secondary growth, which reveals the cyclic nature of the exploration process, was 
189 the highest in the adverse environment (480\% coverage) followed by the control

190 environment (380\%), the slightly nutritive environment (250\%) and the highly

191 nutritive environment (180\%). All comparisons lead to significant differences $\mathrm{P}<0.05$,

192 except control vs. adverse environment (Table 7 and Fig 11 in S1 Appendix). This

193 observation confirms that exploration was slowed down by the presence of nutrients,

194 and that the pulsatile behavior (i.e the exploration of previously explored area) was

195 stimulated by repellents.

196
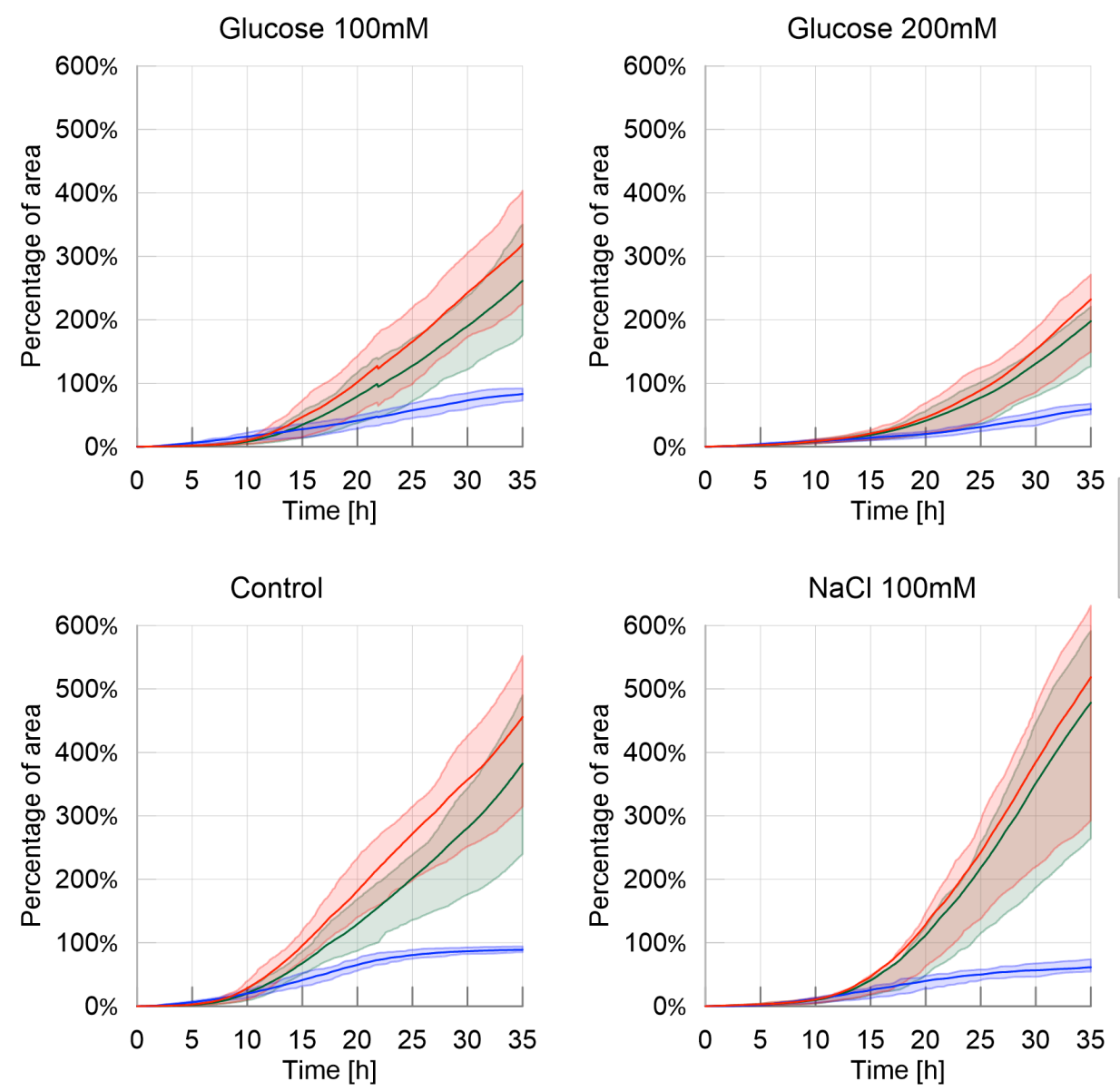

198 Fig 2. Cumulative areas covered by primary growth, refinement and secondary growth -

199 Homogeneous experiments. The solid line corresponds to the average index calculated over the 20 replicates, while the shaded areas correspond to the first and third quartiles of the data. 
202 In accordance with the previous results, the migration rate was higher for the control

203 treatment than for the other treatments (Table 8 and Fig 12 in S1 Appendix: $\mathrm{P}<0.001$

204 for each pairwise comparison). While slime molds exploring the highly nutritive 205 environment were slower than slime molds exploring the slightly nutritive or the 206 adverse environment $(P<0.001$ each), these two showed no significant differences $207(\mathrm{P}>0.05)$.

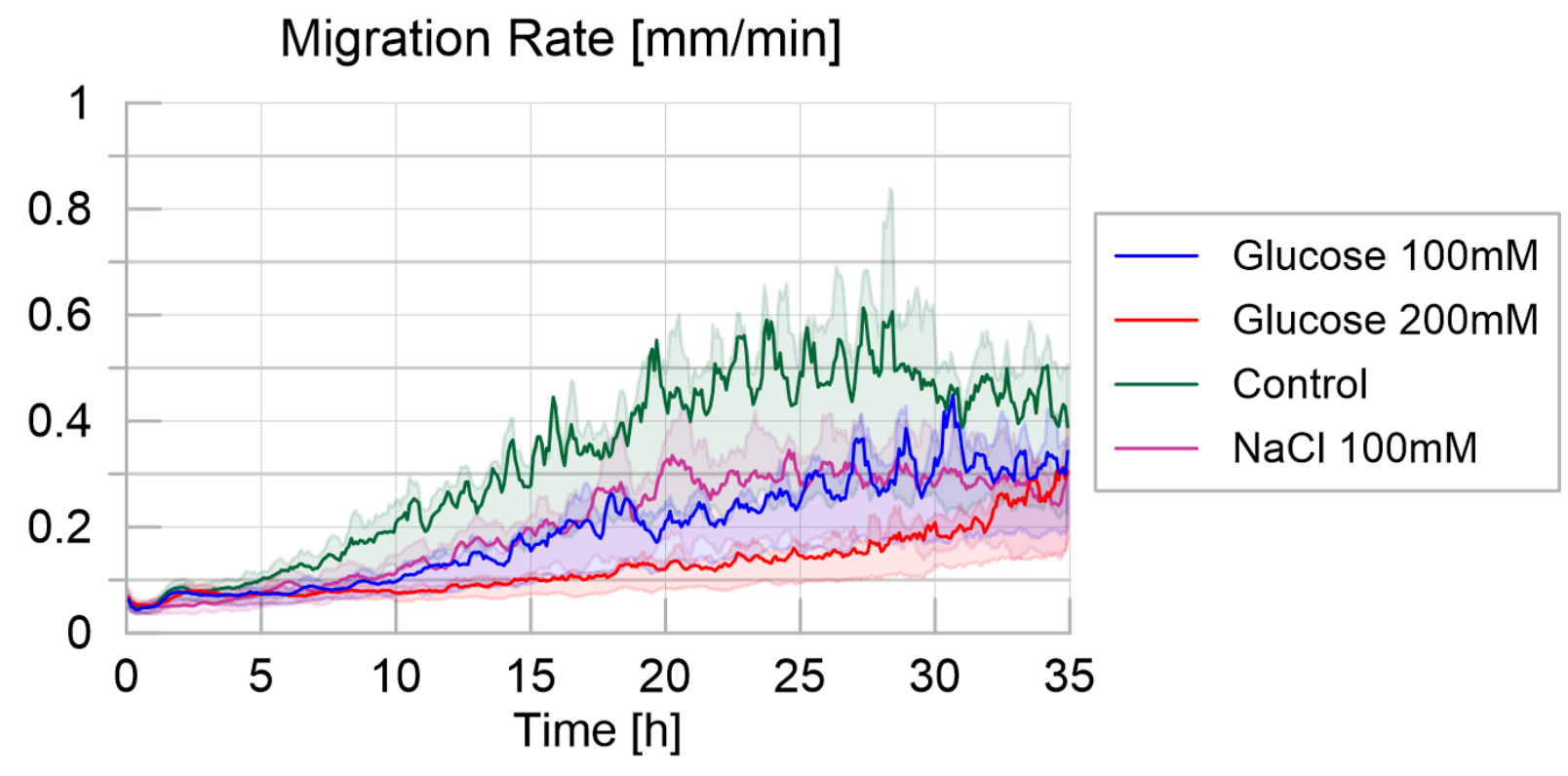

210 Fig 3: Migration rate over time for the four different treatments, defined as the maximum distance

211 between the contours of the slime mold between two consecutive images divided by their time interval

212 (5 minutes apart), measured in millimeters per minute. The solid line corresponds to the average 213 calculated over 20 replicates per treatment, while the shaded areas correspond to the first and third 214 quartiles of the data.

216 The slime molds exploring the adverse environment showed the highest probability 217 to explore a previously explored substrate than the other treatments as shown in Fig 2184 and supplementary materials (Tendency for secondary growth: Table 9 and Fig 13 219 in S1 Appendix: $\mathrm{P}<0.001$ for each pairwise comparison). When exploring a highly 220 nutritive environment, slime molds also displayed a significant positive tendency for 
221 secondary growth ( $P<0.001$ for each pairwise comparison) but significantly less

222 strong than on the adverse environment $(P<0.001)$. For the others treatments, the

223 measured proportion of secondary growth was not different from the expected

224 proportion of secondary growth, indicating that the slime molds did not avoid

225 previously explored substrate and explored randomly (Fig 13 in S1 Appendix). The

226 peaks observed within the first 5 hours of the experiment correspond to an isotropic

227 extension immediately followed by a refinement process that occurred before the

228 slime mold started to explore continuously its environment. This behavior is often

229 observed when a slime mold is introduced in a new environment and is referred as

230 "contemplative" (49) i.e. the slime mold migrates, retracts and moves again. The

231 peak was larger for adverse environment.

232 

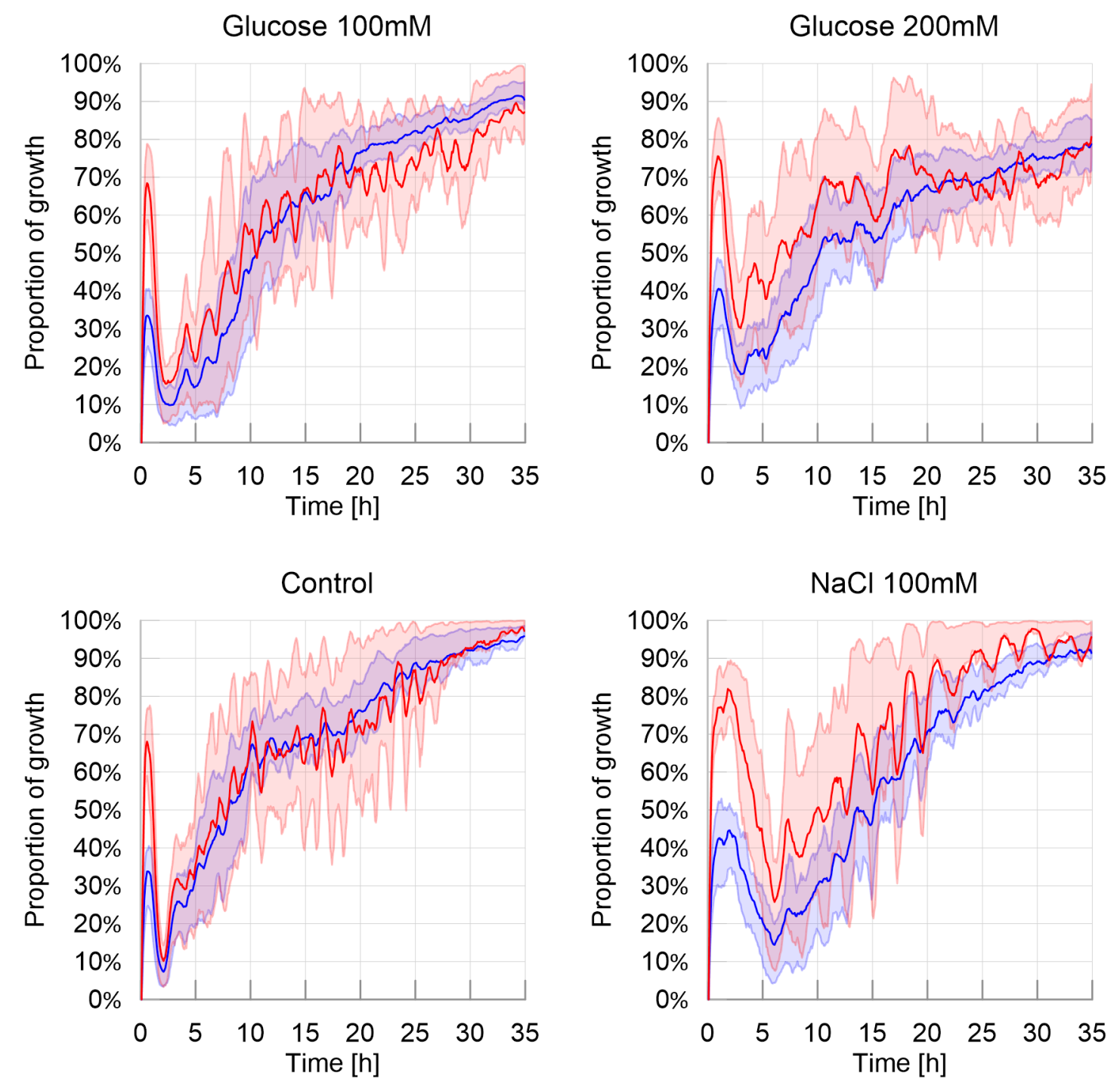

Expected proportion of secondary growth Interquartile range (expected growth)
Measured proportion of secondary growth Interquartile range (measured growth)

234 Fig 4: Ratio of secondary growth: observed and expected proportion of secondary growth. The solid

235 line corresponds to the average calculated over 20 replicates per treatment, while the shaded areas

236 correspond to the first and third quartiles of the data.

238 We then analyzed the evolution of the shape of the slime molds contour. Note that

239 the experimental set ups in which slime molds were placed exhibited radial 240 symmetry. Hence, no preferential expansion direction was expected. We thus 241 focused on contour shape, not orientation. 
242 As expected, circularity was initially one in all tests (circular slime mold spot), and

243 increased over time as the contour shape departed from a circle (Fig 2 in $\mathrm{S} 1$

244 Appendix). In the control and nutritive environments, circularity remained between

2451.05 and 1.10, whereas it fluctuated between 1.05 and 1.30 in the adverse

246 environment. This observation suggests that, in an adverse environment, slime

247 molds explored the petri dish by spreading and thinning over larger areas than in the

248 other environments, which led to shape changes and a decrease of slime mold

249 circularity. However fluctuations among the 20 replicates were too high to identify

250 any trend in the evolution of slime mold circularity.

252 The eccentricity index was initially close to zero (circular cell) and increased up to almost 0.8 over time, with important fluctuations in all the treatments (Fig 3 in S1

254 Appendix). Eccentricity is an indicator of the number of pseudopodia. But a noneccentric convex hull can enclose non-circular contours of slime mold, since

256 pseudopodia can develop in a symmetric fashion. That is why no major difference 257 was noted between the treatments. This result highlights the absence of preferred expansion direction in symmetric, homogeneous environments.

259 Solidity decreased with the emergence of pseudopodia, since slime mold branching 260 disrupted the initially convex shape of the slime mold (Fig 5). In the control 261 environment, in which the exploration rate was the highest, the decrease of solidity 262 of the slime mold area was the highest (and the fastest) decreasing from 1 to 0.3 and 263 then becoming relatively stable, with fluctuations of +/- 0.05 (Table 10 and Fig 14 in 264 S1 Appendix; $\mathrm{P}<0.01$ for all paired comparisons except adverse environment vs. 265 slightly nutritive environment, where $\mathrm{P}>0.05$ ). Slower exploration resulted in a slower 266 and steadier loss of solidity as observed in the nutritive and adverse environments. 
267 The highly nutritive environment yielded the highest solidity at the end of the experiment (0.6), which confirmed that the presence of glucose slowed down

269 exploration.

270

271

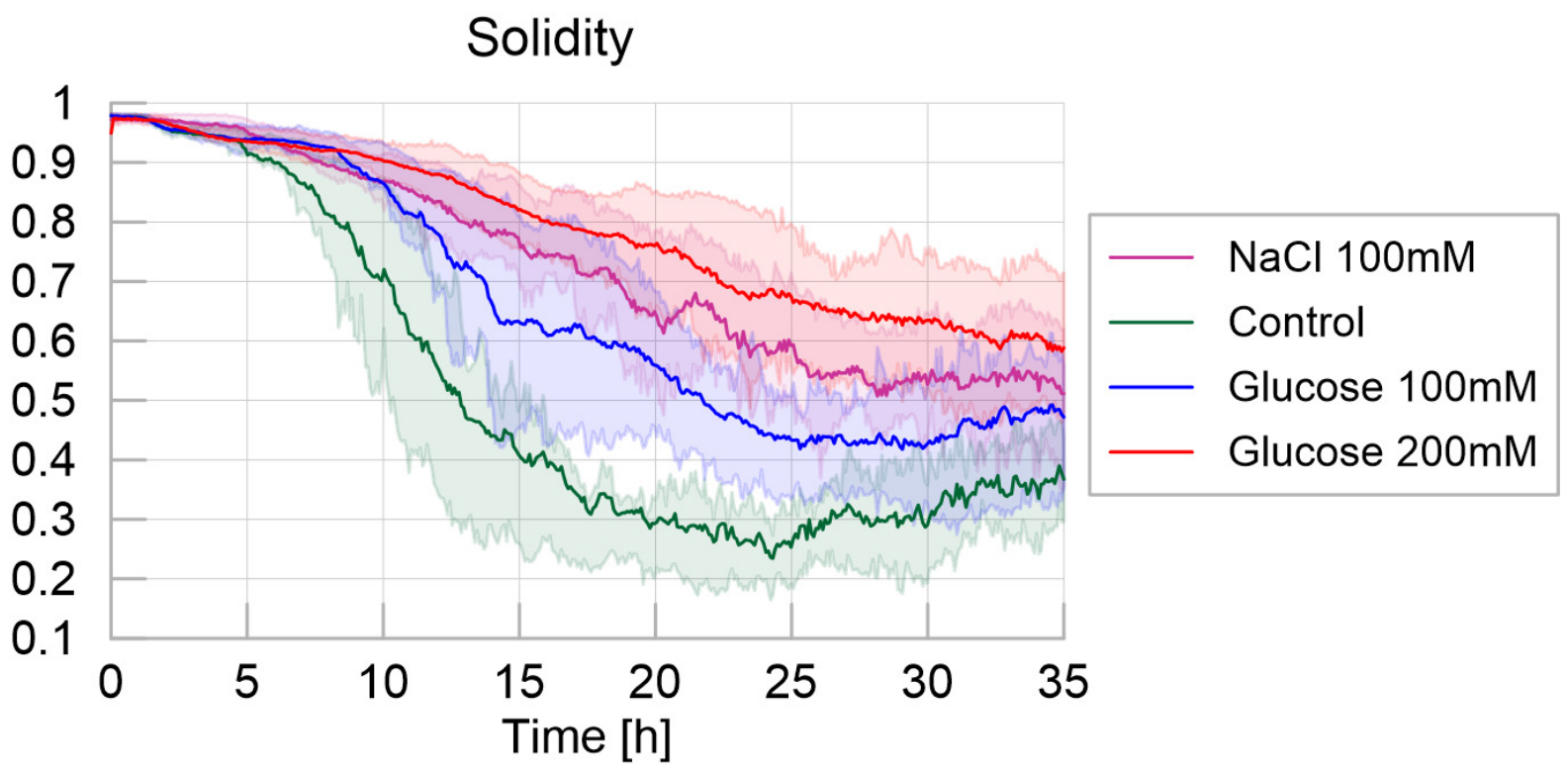

272 Fig 5. Solidity - Homogeneous experiments. The solid line corresponds to the average index

273 calculated over the 20 replicates, while the shaded areas correspond to the first and third quartiles of

274 the data.

275

276 We next focused on the number of clusters, corresponding to the number of

277 pseudopodia (Fig 6). Initially the slime molds stretched as a single cluster. Once

278 mucus started to be apparent, slime molds usually divided up into several clusters,

279 and started the active exploration phase. In highly nutritive and adverse

280 environments, the number of clusters over time was lower than in the other two

281 treatments (new pseudopod number: Table 11 and Fig 15 in S1 Appendix, and P <

2820.01 for all paired comparisons except for adverse environment vs. highly nutritive

283 environment). This observation confirmed that the presence of concentrated

284 nutrients slowed down the exploration, and that the presence of repellents triggered 
bioRxiv preprint doi: https://doi.org/10.1101/622662; this version posted April 29, 2019. The copyright holder for this preprint (which was not certified by peer review) is the author/funder, who has granted bioRxiv a license to display the preprint in perpetuity. It is made available under aCC-BY 4.0 International license.
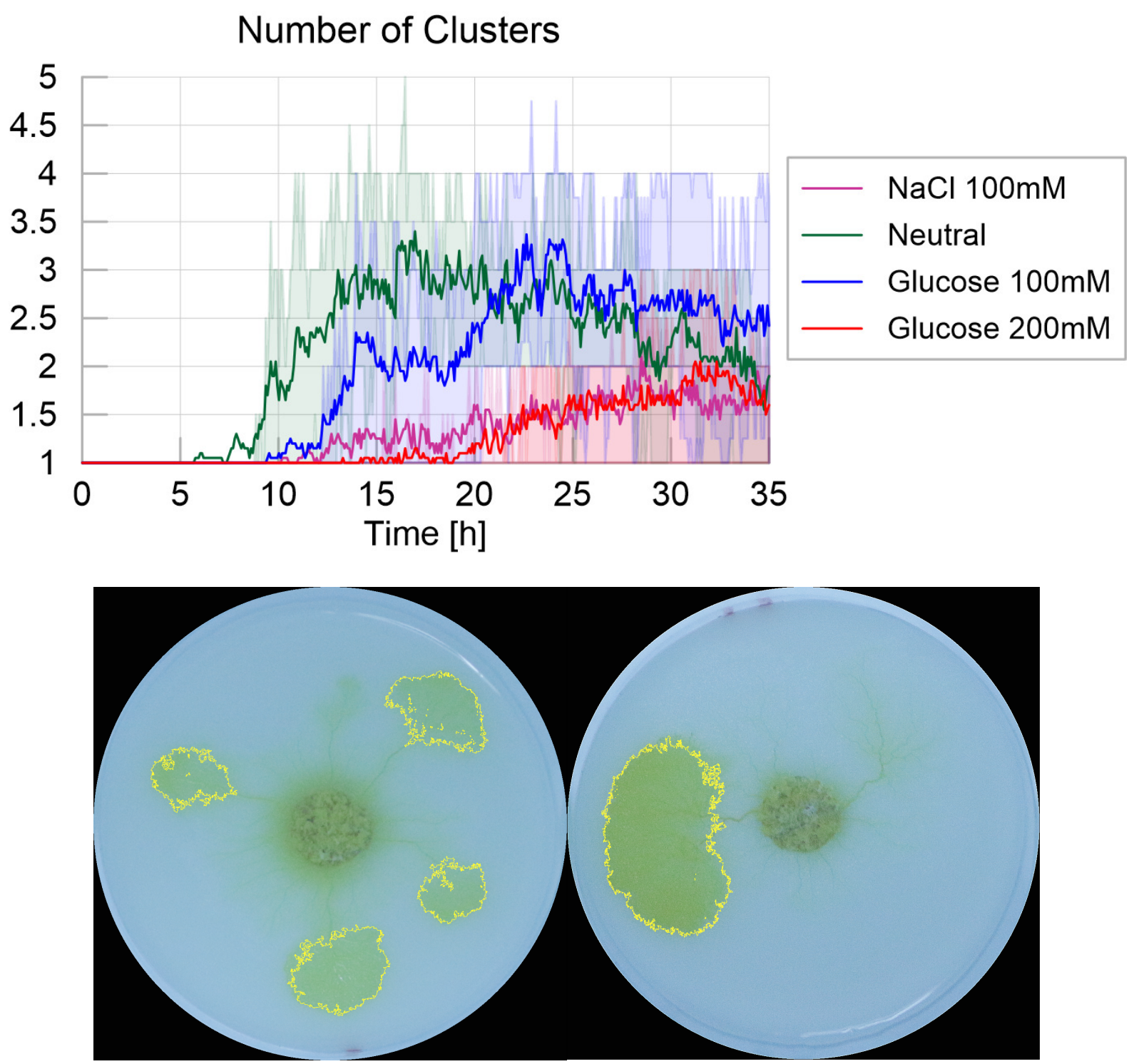

290 Fig 6. Number of clusters - Homogeneous experiments. The solid line corresponds to the average

291 index calculated over the 20 replicates, while the shaded areas correspond to the first and third 292 quartiles of the data. Pictures show examples of clusters for the Control environment (left) and $293200 \mathrm{mM}$ Glucose environment (right). 


\section{2) Spot experiments}

298 In the spot experiments, we studied the influence of discrete distributions of nutrients

299 and repellents on exploration dynamics. When looking at the evolution of slime mold,

300 mucus and unexplored substrate over time (Fig 7), we only observed marginal

301 difference among the treatments, which all exhibited similar patterns of exploration,

302 e.g. similar percentage of non-explored area and similar mucus accumulation. The

303 presence of an adverse spot only delayed the appearance of the first pseudopod

304 (first movement: Table 12 and Fig 15 in S1 Appendix, $\mathrm{P}<0.05)$ but not the first

305 appearance of mucus (first appearance of mucus: Table 15 in S1 Appendix, not

306 significant). The only noticeable differences lie in the surface reached at the end of

307 the experiment: slime molds that were offered a highly nutritive spot grew larger

308 (final surface: Table 14 and Fig 17 in S1 Appendix; $\mathrm{P}<0.01$ ). By contrast, the

309 surface covered by mucus was lower (mucus final surface: Table 17 and Fig 18 in

310 S1 Appendix; $\mathrm{P}<0.01)$ than slime molds that were offered a slightly nutritive spot. In

311 comparison with the experiments conducted in homogeneous environments, we did

312 not observe any expansion/refinement cycles in the spot experiments, meaning that

313 slime mold spread steadily towards the food source despite the presence of an

314 obstacle on the way. 

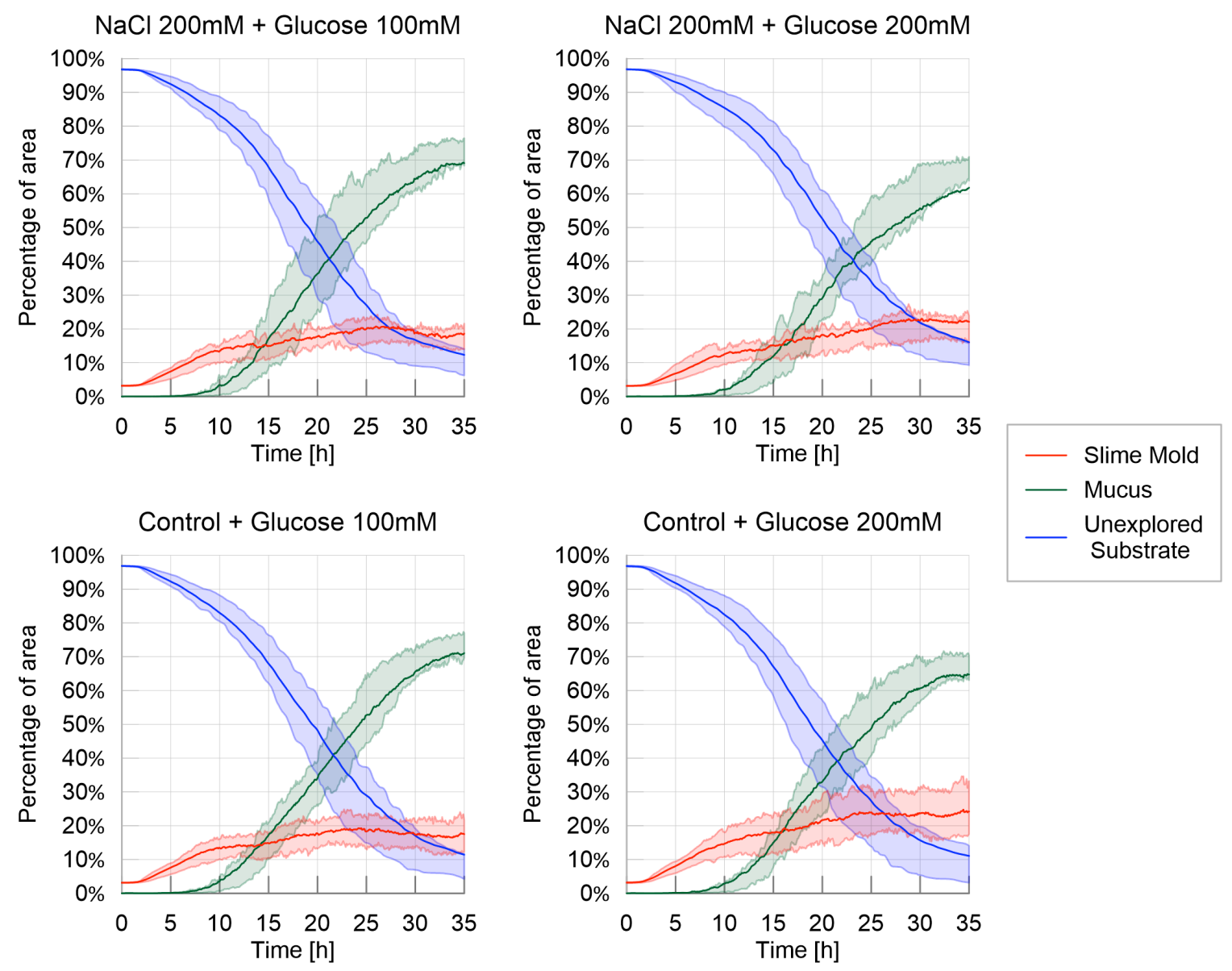

Fig 7. Fraction of area covered by each entity (slime mold, mucus, unexplored substrate) - Spot

318 experiments. The solid line corresponds to the average index calculated over the 20 replicates, while

319 the shaded areas correspond to the first and third quartiles of the data

321 The exploration behavior in the spot experiments was similar to that observed in the

322 control environment in homogeneous experiments as observed in Fig 8, which

323 includes the average percentage of unexplored area for the homogeneous and spot

324 experiments shown in Fig 1 and Fig 7 respectively. This suggests that the spatial 325 exploration of slime mold depended mostly on the substrate and not on the 326 geometric distribution of the nutritive and adverse stimuli. 

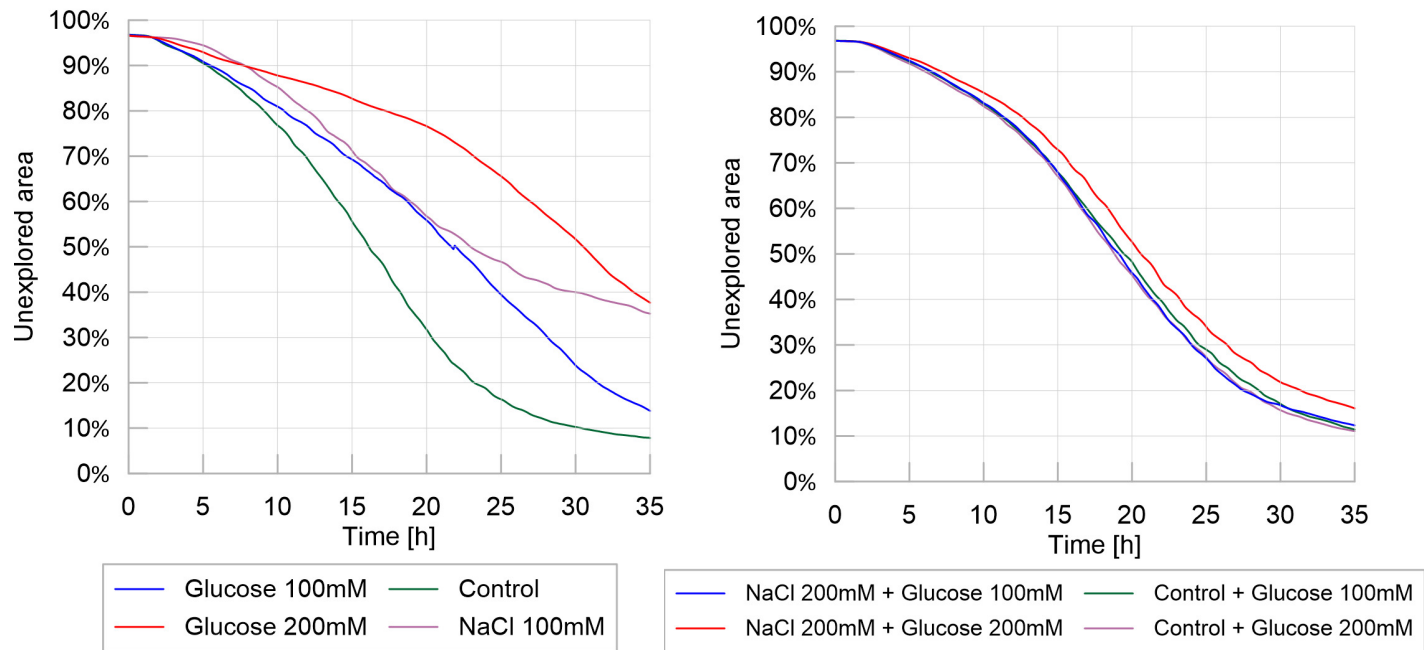

329 Fig 8. Exploration behavior: homogeneous experiments (left) vs. spot experiments (right). Percentage

330 of unexplored area over time. Mean values of over 20 replicates for each different treatment.

332 The cumulative areas covered by secondary growth for the spot experiments (Fig 9)

333 were also similar for all treatments (Table 18 in S1 Appendix, $\mathrm{P}>0.05$ ), suggesting

334 again, that isolated spots with nutritive or adverse stimuli did not alter the overall exploration of slime molds when growing on the same, control, substrate. 

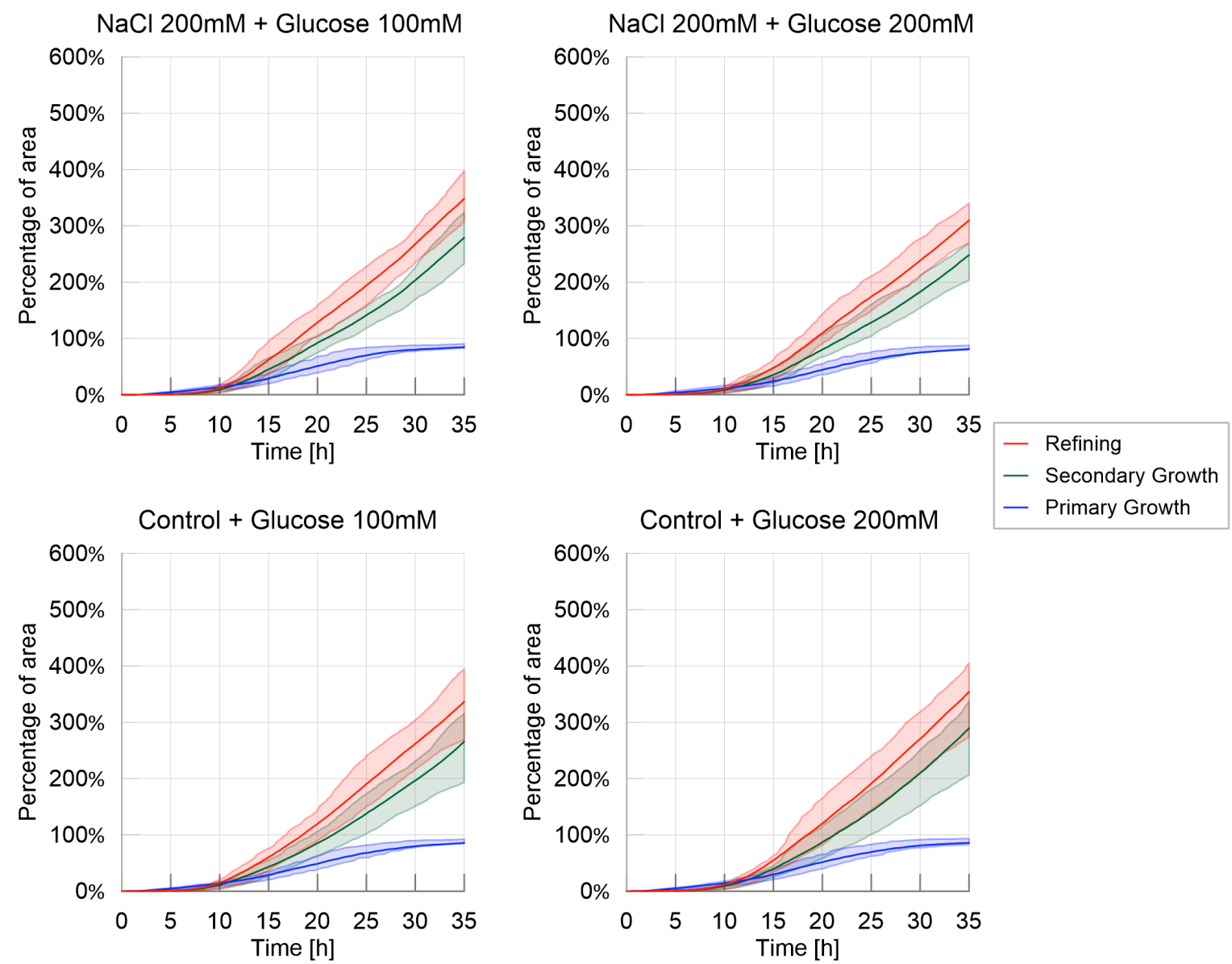

338 Fig 9. Cumulative areas covered by primary growth, refinement and secondary growth - Spot 339 experiments. The solid line corresponds to the average index calculated over the 20 replicates, while 340 the shaded areas correspond to the first and third quartiles of the data

342 The trends of migration rate, as shown in Fig 10, show that slime molds were not

343 affected by the difference between treatments, showing only a slight effect of the 344 concentration of the food spot $(\mathrm{P}<0.05)$, as shown on Table 19 in $\mathrm{S} 1$ Appendix. This 345 effect showed that the migration rate was slightly superior when slime molds were 346 offered a higher than a lower nutritive spot. 


\section{Migration Rate $[\mathrm{mm} / \mathrm{min}]$}

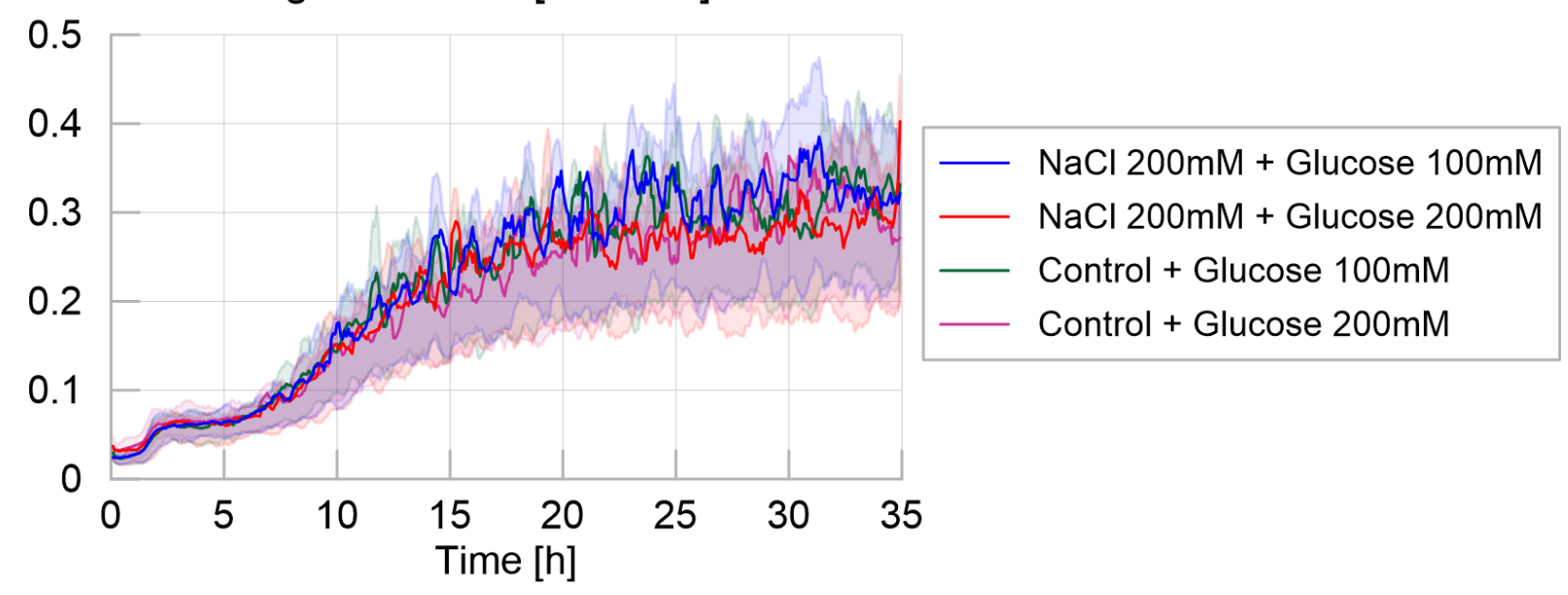

348 Fig 10: Migration rate over time for the four different treatments, defined as the maximum distance 349 between the contours of the slime mold between two consecutive images, divided by their time 350 interval (5 minutes apart), measured in millimeters per minute. The solid line corresponds to the 351 average calculated over 20 replicates per treatment, while the shaded areas correspond to the first 352 and third quartiles of the data.

354 Similarly, looking at the predilection of slime molds to grow towards mucus 355 (secondary growth), as shown in Fig 11, no significant differences were observed between treatments (Table 19 in S1 Appendix), which suggests that the growth type 357 is not influenced by the existence or concentration of discrete attracting/repelling 358 spots. 

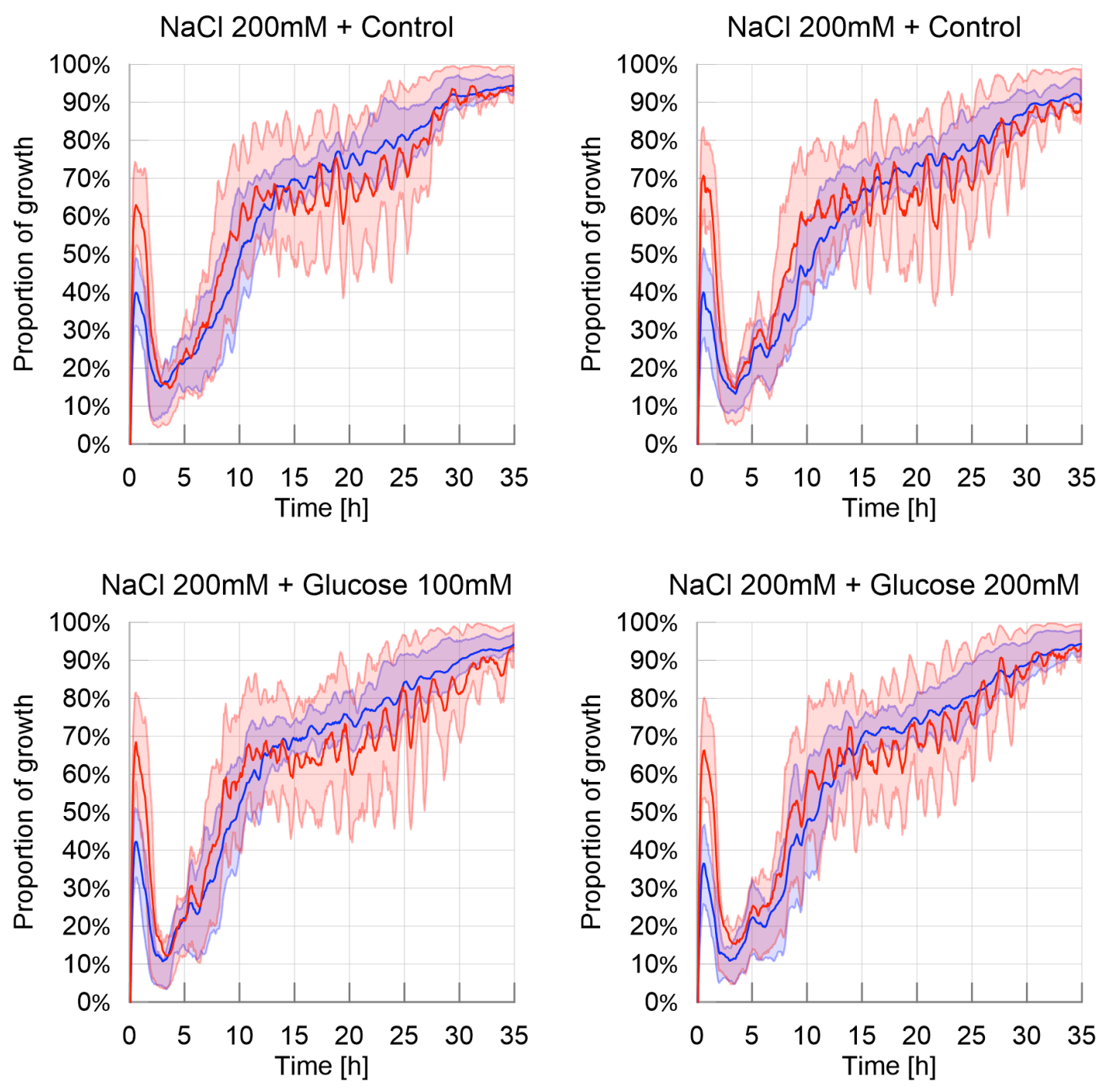

Expected proportion of secondary growth Interquartile range (expected growth)
Measured proportion of secondary growth Interquartile range (Measured growth)

361 Fig 11: Probability of secondary growth: observed and expected proportion of secondary growth. The solid line corresponds to the average calculated over 20 replicates per treatment, while the shaded areas correspond to the first and third quartiles of the data.

365 The results obtained for the four different shape indexes (Fig 4 in S1 Appendix) for

366 the spot experiments support the hypothesis that discrete spots of nutrients or repellents did not affect the overall expansion dynamics and exploration cycles. This interpretation is confirmed by the average number of pseudopods, which was found 
369 to be correlated to the formation of mucus during the exploration phase: in all the spot experiments, the number of clusters increases from 1 to 2.5 within around 12

371 hours, to reach a plateau afterwards. In other words, less exploration cycles were

372 observed in non homogenous environments, yielding less pseudopodia.

374 The evolution of the shortest distance from the slime mold cell to the glucose spot is shown in Fig 12, which can be viewed as a "survival" plot, displaying the proportion of the replicate $(P)$ in which slime mold has not reached the nutritive spot at a given time. Both increasing the concentration of nutrient in the spot (from slightly nutritive to highly nutritive) and adding an aversive spot increased the time to reach the food patch by decreasing the probability to reach it (time to food patch: Table 22 and Fig
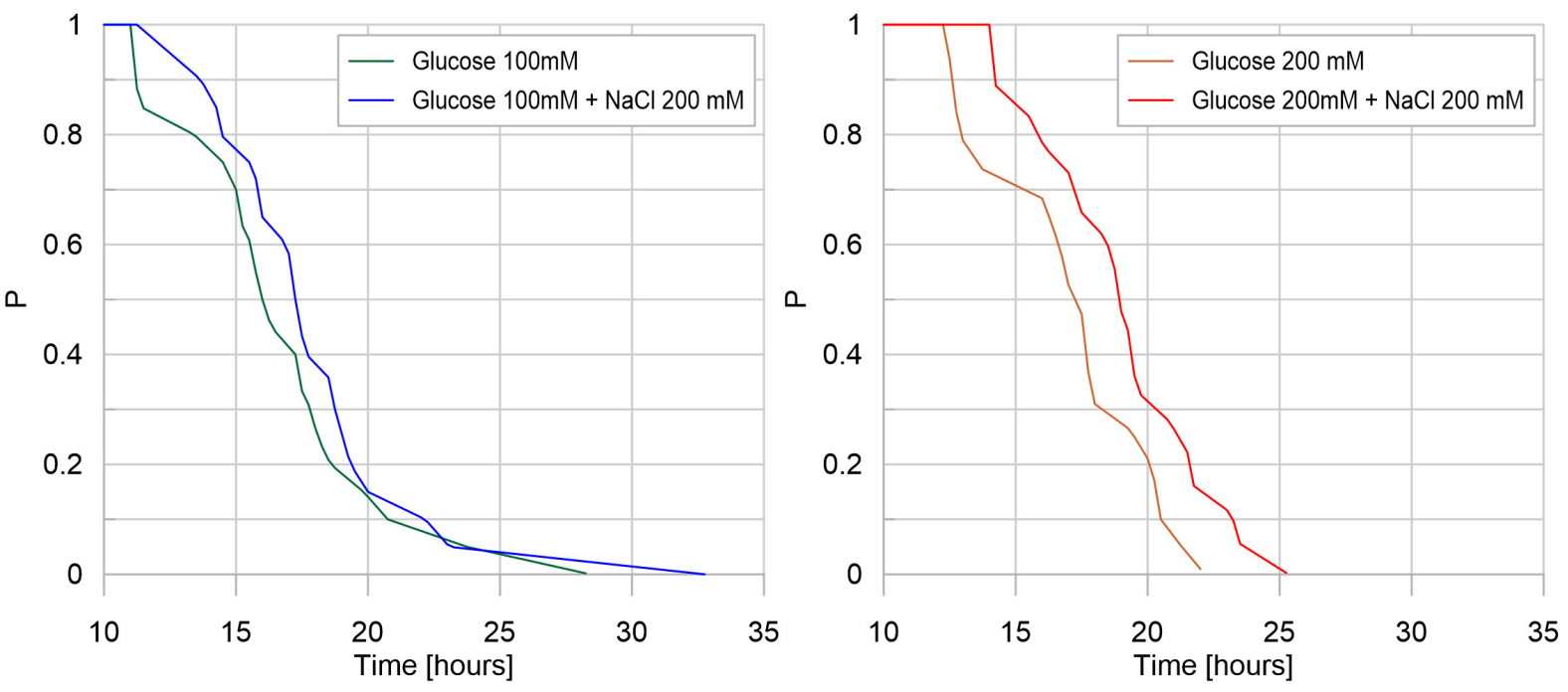

383 Fig 12. Survival plot: glucose concentration effect. For each treatment, on the vertical axis, the value $384 \mathrm{P}$ corresponds to the fraction of replicates that have not reached the glucose spot at a given time. For a representative number of replicates, $\mathrm{P}$ is the probability that glucose has not been reached by slime mold at a given time, for a specific treatment. On the horizontal axis, each value of time corresponds to the average time it takes for a certain fraction of slime mold $(\mathrm{P})$ to reach the glucose spot. 


\section{Discussion}

390

391 Exploration in slime molds involves two different processes: area extension and 392 movement (27). Slime molds locomotion and morphogenesis depend on the 393 response of the organism to the environmental conditions. In the present paper, we 394 show that distributions of nutritive and aversive cues affected drastically the 395 exploration pattern of slime molds.

397 The typical exploration behavior of a slime mold (control condition) started by a stretching period where the slime molds grew uniformly in all directions for 10 hours.

399 Then, the contour of slime mold lost circularity when the first pseudopodia appeared, 400 which also corresponds to the first occurrence of mucus. This phenomenon is typical 401 of the directed digitated growth, or branching phase, described by numerous authors $402(27,51,59-61)$. Slime molds developed multiple pseudopodia and did not exhibit any preferential exploration orientation. At the end of the experiment, almost all the arena

404 was explored by the slime mold. Thus the exploration was characterized by three 405 phases: (i) Primary growth only, in the quasi-absence of mucus (5-10 hours); (ii) 406 Combination of primary and secondary growth; (iii) Secondary growth only, when the slime mold stops exploring new substrate areas.

408

\section{1) Homogeneous distribution of nutritive cues}

411 First, we noted that an environment containing a uniform distribution of nutrients 412 slowed down the exploration of slime mold, mainly by delaying secondary growth 413 and increasing the period of the pulsatile exploration/refinement movements. The 
414 area not explored by the slime molds was 3 times larger (respectively 7 times larger)

415 than in the environment deprived of nutrient (control case) for a slightly nutritive

416 (respectively highly nutritive) environment. The exploration rate was almost linear for

417 highly nutritive environments, while for other treatments, the area covered by the

418 slime molds reached a plateau after a period of stretching, which indicates

419 secondary growth and slime mold displacement. This means that slime molds that

420 explored nutritive environments never exhibited a Phase (iii) in their exploration

421 pattern.

422 Second, on substrates with higher nutrient concentrations, the slime molds grew in a

423 more compact fashion, i.e. slime molds presented the highest solidity index and the

424 lowest number of pseudopodia (clusters). Additionally, the appearance of mucus,

425 which indicates that the slime mold was withdrawing, occurred much later in nutritive

426 environments. As glucose is only aversive when only above $300 \mathrm{mM}$ (54), our results

427 suggest that nutritive media depressed migration due to feeding behavior. This

428 allows the organism to remain at a site until nutrients are exhausted $(54,62,63)$. In

429 previous studies, it was shown that the area of substrate covered increases when

430 slime mold responds to nutrient dilution $(54,64)$. Here, we confirmed these

431 observations and noted that slime mold tended to migrate and grow faster on

432 substrates with the lowest concentration of nutrients, thus maximizing nutrient intake

433 and optimizing the trade-off between nutrient foraging and nutrient intake.

\section{2) Homogeneous distribution of aversive cues}

437 The aversion of slime mold to salt manifested itself through longer contemplative 438 behavior, delayed primary growth and a higher probability to crawl on previously 
439 explored surface. In addition, the slime molds grew more compactly and with less

440 pseudopods. This suggests that slime molds were actively avoiding contact with the

441 aversive surface $(44,49)$. In the absence of cell walls, slime mold has no other

442 protection from the environment than mucus. Indeed, in bacteria for instance, mucus

443 is used as protective barrier for the cells against harsh external conditions (65). In

444 slime molds, the extracellular mucus excreted by the slime molds can have different

445 roles: hydrophilic shield to prevent water loss (66), a lubricating surface over which

446 the slime molds can easily crawl (67), a defensive coat to protect against invasion by

447 foreign materials and organisms $(66,68)$, an aid to phagocytosis $(69)$, a surface that

448 promotes ion-exchange (66) and has externalized spatial memory that helps

449 navigation in unknown environments $(26,36,53)$. Here, we can add a new function for

450 the mucus i.e. a buffer to move in adverse environment.

451

452 3) Non-Homogeneous distribution of aversive and non aversive cues

454 In the spot experiments, pulsatile movements were limited and slime molds 455 responded in the same way regardless of the concentration of glucose used as attractant and regardless of the presence of a salt spot on the way to the glucose spot. Slime molds grew in a more compact fashion, i.e. slime molds presented the

458 highest solidity index and the lowest number of pseudopodia (clusters). Additionally, 459 the evolution of the areas covered by slime mold and mucus over time indicates that 460 the response of slime molds to heterogeneous environments was similar to that in 461 the control case. This result suggests that in the spot experiments, the exploration 462 behavior of slime mold is mostly controlled by the substrate. Our observation 463 confirms that salt reception can be affected by the presence of sugars (46). The 
464 authors in (46) showed that the "apparent" enthalpy change accompanying salt perception decreases with increase of sugar concentration.

466

467 The proposed image analysis program allows extracting information on expansion 468 rates, geometric changes and probability of occupancy. Ongoing developments aim 469 to acquire high quality images of slime mold exploration tests and to expand the 470 code's capabilities to extract topological information on the networks formed by slime 471 molds. Possible applications of the code go beyond the study of slime molds. For 472 instance, the analysis of successive images of an ant nest could allow detecting the 473 generation of any rhythmic activity. Trajectories of individuals within a flock could 474 also be described to understand whether or not members of the flock can inform and 475 influence the travel direction of other individuals. Such a finding would allow 476 understanding how group decisions are made among gregarious species. The 477 cluster identification and shape recognition program could be used to differentiate 478 modes of gene expression or to recognize objects. Object identification is an 479 important pillar to explain associative memory or to track species interactions in an ecosystem. The tools and approach presented here are thus applicable to any problem of network dynamics or pattern recognition.

482

\section{Methods}

\section{1) Species}

Physarum Polycephalum, also known as the true slime mold, belongs to the 486 Amoebozoa, the sister group to fungi and animals (50). Slime molds are found on 487 organic substrates like tree bark or forest soil where they feed on microorganisms 488 such as bacteria or fungi (50). The vegetative morph of $P$. polycephalum, the 
489 plasmodium, is a vast multinucleate cell that can grow to cover up to a few square

490 meters and crawl at speeds from 0.1 to few centimeters per hour $(29,30)$. When

491 hygrometry and food availability decrease, the plasmodium turns into an encysted

492 resting stage made of desiccated spherules called sclerotium (29).

\section{2) Rearing conditions}

494 Experiments were initiated with a total of 10 sclerotia per strain (Southern Biological, 495 Victoria, Australia). We cultivated slime molds on a 1\% agar medium with rolled oat 496 flakes, slime molds were fed every day and the medium was replaced daily. Slime 497 molds were 2 weeks old when the experiment started. All experiments were carried 498 out in the dark at $25^{\circ} \mathrm{C}$ temperature and $70 \%$ humidity, and ran for $35 \mathrm{~h}$. Pictures 499 were taken every 5 min with a Canon 70D digital camera.

\section{3) Experimental setup}

501 Initially we monitored the exploration movement evoked in slime molds in a 502 homogeneous environment. Each slime mold was placed in the center of a circular 503 arena $(14.5 \mathrm{~cm}$ in diameter) with a layer of agar (1\% in water) mixed with non504 nutritive cellulose (5\%). Adding cellulose to the agar mix proved to be useful to 505 obtain a homogeneous pigmentation and to enhance the color contrast between the 506 substrate and slime mold, therefore improving the identification process. A circular 507 hole $(2.5 \mathrm{~cm}$ in diameter) was punched and replaced with a circular slime mold of the 508 same size sitting on oat. In the first and second treatments (nutritive environments) 509 we added glucose $(100 \mathrm{mM}$ or $200 \mathrm{mM})$ to the medium. In the third treatment 510 (adverse environment), we added a known repellent ( $\mathrm{NaCl} 100 \mathrm{mM}(51))$ to the 511 medium. Lastly, in the fourth treatment, the medium remained unchanged (neutral 512 environment i.e. control treatment). 
514 Subsequently, to investigate how chemotaxis modified the exploration behavior, we

515 introduced discrete spots of attractants/repellants within a neutral substrate made of

516 plain agar. In these so-called "spot experiments", we followed a procedure similar to

517 that for the homogeneous environments. A circular slime mold $(2.5 \mathrm{~cm}$ in diameter $)$

518 was placed diametrically opposite to a glucose (attractant) spot of same size placed

$5194.5 \mathrm{~cm}$ away. In some of the treatments, a salt $(\mathrm{NaCl} 200 \mathrm{mM}$, repellent) spot was

520 added at the center of the petri dish. A total of 4 different treatments were tested: the

521 first and the second with a single spot of glucose at concentrations of $100 \mathrm{mM}$ and

522 200mM respectively, the third and fourth keeping the glucose spots with the same

523 concentrations and adding a $\mathrm{NaCl} 200 \mathrm{mM}$ spot at the center of the dish.

524

525 All slime molds were fed just before the experiment so we assumed that they were in

526 the same physiological state. The experiment consisted of a total of 8 different

527 treatments. We replicated the experiment 20 times for each treatment and monitored

528 each arena for 35 hours taking time-lapse photographs every 5 minutes. Fig 13

529 shows the experimental set-up for homogeneous environments (left) and discrete

530 distributions of attractants/repellants (right).
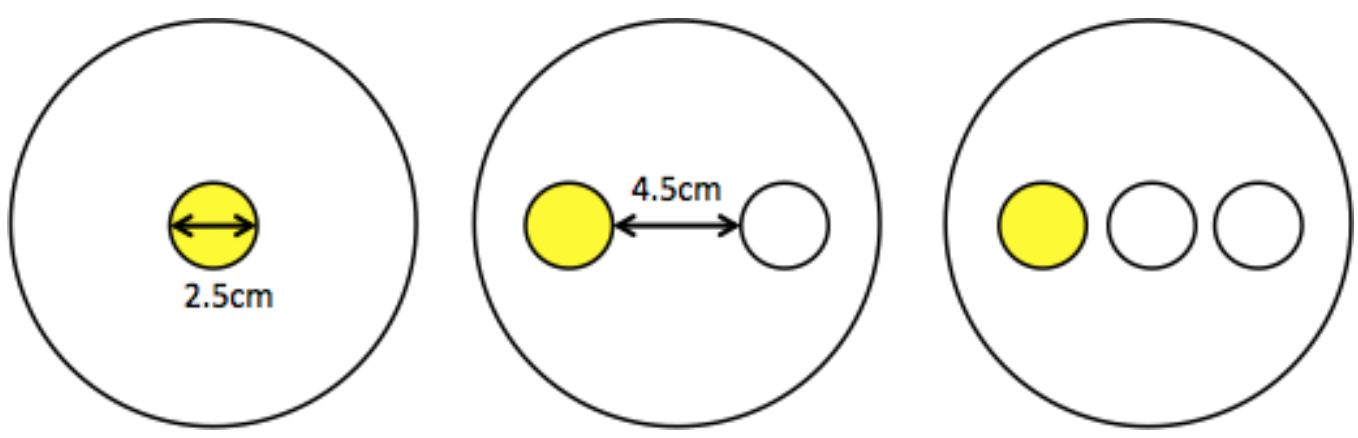

532 Fig 13: Experimental set-ups for homogenous environment and spot experiment 


\section{4) Image Processing}

535 Time-lapse images were taken every 5 minutes for a total of 420 pictures for each

536 replicate. The images acquired initially belong to the RGB color space and their size

537 was 1200 by 1200 pixels. Image analysis followed three main steps. First, the edge

538 of the petri dish was identified by fitting its border to a circle of known diameter.

539 Second, the image was segmented using the clustering algorithm k-means (52),

540 which was applied to the images converted into the $a b^{*}$ color space, which is the

541 CLAB space without the $L^{*}$ (lighting) component; this choice corresponds to the

542 robustness of this color space against changes of lighting conditions between

543 images. By the end of this step, all the pixels inside petri dish, e.g. the region of

544 interest (ROI), are identified as either slime mold or not-slime mold.

545

546 After distinguishing slime mold and non-slime mold areas, we identified the mucus

547 by using a subtraction method. The mucus is left by slime after refinement; this

548 substance acts as a marker present on already explored areas of the domain,

549 serving as an external memory to the slime mold $(36,53)$. The fact that this mucus is

550 transparent makes it very challenging to identify by sole color analysis. We thus

551 trinarized the image based on the history of a given pixel, since a pixel that is

552 classified as non-slime-mold at the current image will necessarily contain mucus if it

553 has ever been classified as slime mold in any of the previous images. Conversely, a

554 non-slime-mold pixel will be classified as unexplored substrate if it has never hosted

555 slime mold up to that point in time.

557 The change of class from unexplored substrate to slime mold, defined as primary 558 growth, means that a new sprout of the slime mold reaches a point in space that it 
559 had never explored before. Similarly, secondary growth is defined as the change

560 from mucus to slime mold, meaning that the slime mold is revisiting an already

561 explored location. Lastly, if the slime mold recedes from a point, e.g. a pixel goes

562 from slime mold to non-slime mold, it becomes mucus, and the process is defined as

563 refinement. By the end of these three steps, the images have been trinarized,

564 meaning that every pixel is classified as slime mold, unexplored substrate, or mucus

565 (and the points outside the ROI are disregarded). Two videos are provided to the

566 reader as supplementary material, S2 and S3 show one of the replicates as original

567 images and trinarized images after identification respectively.

568

569 5) Image Analysis in Space and Time

570

571 After completing the trinarization, we calculated several indexes to characterize

572 slime mold geometry dynamics. We averaged the indexes over the 20 replicates of

573 each treatment in order to obtain statistically representative measures, and we

574 plotted them over time.

575

576 In order to quantify the differences of slime mold spreading dynamics on distinct

577 substrates, we first calculated the fraction of the petri dish area covered by slime

578 mold, mucus and unexplored substrate over time. The total area, the lighting

579 conditions and the test duration were the same for all treatments, both in the

580 homogeneous and spot experiments. Note that glucose only provides energy to

581 slime mold, which is not gaining significant mass during the experiments (54). In

582 other words, slime mold is changing its area by mostly by stretching and contracting,

583 therefore changing its area density. 
585 In order to gain further insights about the exploration process we then computed the

586 cumulative area of primary growth, refinement, and secondary growth over the full

587 period of the experiments comparing two consecutive images at the time. The

588 cumulative area covered by primary growth is indicative of the total area of

589 exploration, therefore it is always smaller or equal to the total area of the dish. The

590 cumulative area covered by secondary growth indicates whether slime mold

591 expansion is monotonic (dominated by primary growth) which results in a smaller

592 magnitude, or cyclic (secondary growth dominated, with pulsatile movements) which

593 results in a larger magnitude. The cumulative area covered by refinement indicates

594 slime mold density changes. Within a given time interval, if the area covered by

595 primary plus secondary growth equals that covered by refinement, then slime mold

596 keeps the same density, whereas if it is superior, the slime mold stretches (e.g.

597 density decreases). If secondary growth is negligible and if the area covered by

598 primary growth equals the area covered by refinement, then slime mold displaces

599 mass.

600

601 We next measured the migration rate. To this aim, for two consecutive images, we

602 measured the distance from each pixel where growth occurred (both primary and

603 secondary) to the closest pixel classified as slime mold in the previous image. This

604 distance represents the extent of growth from one image to the next. We calculated

605 the migration rate as the ratio between the maximum distance traveled and the time

606 interval between two images (5 min). This maximum distance traveled was then

607 used to delineate the region explored by slime mold within the 5 min interval. In other

608 words, we defined an area of interest as the contour of the slime mold with an offset 
609 corresponding to the maximum distance traveled (see Fig 1 in S1 Appendix for more

610 details).

611

612 We estimated the fraction of secondary growth as the ratio between the number of

613 pixels changing from mucus to slime mold and the total number of pixels in the

614 region of interest. We then calculated the fraction area of "expected secondary

615 growth", which would have occurred if secondary growth had happened randomly. If

616 the measured secondary growth fraction is higher (respectively, lower) than the

617 expected one, this means that slime mold has a bias towards mucus (respectively,

618 unexplored substrate).

620 Additionally, we computed four shape parameters indicative of the contour of slime

621 mold: circularity, eccentricity, solidity and number of clusters. Circularity (C) is

622 defined as:

$$
C=P^{2} / 4 \pi A
$$

623 Where $\mathrm{P}$ and $\mathrm{A}$ are the perimeter and area of the shape of slime mold at a given

624 time; this index is equal to one when the contour of slime mold is circular, and

625 increases as the shape deviates from the circle. Eccentricity $(E)$ is calculated as the

626 ratio between the distance between the foci and the major axis length, as follows:

$$
E=\sqrt{1-\left(\frac{b}{a}\right)^{2}}
$$

627 In which $a$ and $b$ are the lengths of the major and minor axes, respectively. When $\mathrm{E}$ 628 is equal to zero, the contour is a circle; when $E$ is equal to one, the contour is 629 degenerated into a line. Solidity (S) is the ratio between the area of the slime mold 630 contour and the area of its convex hull, e.g. the smallest convex polygon that 
631 encloses all the slime mold pixels. $S$ is equal to unity when the contour shape is

632 convex. Lastly, we measured the number of clusters by performing an "erosion"

633 operation along the contour of the slime mold, consisting in removing the veins that

634 connect the regions of high concentration of slime mold. After this erosion process,

635 only the clusters of high concentration of slime mold remained, which provided the

636 number of pseudopodia at the given image.

638 For the spot experiments, we also determined the distance from the slime mold to

639 the glucose spot at every time step. This distance was calculated as the minimum

640 distance between the contour of the slime mold and the perimeter of the glucose

641 circular spot. The evolution of the distance to glucose over time was analyzed in a 642 way similar to a survival analysis, as described below.

\section{5) Statistics}

646 The full description of the statistics is provided as part of the supplementary 647 information; Appendix S1 includes the results of the complete statistical analyses, 648 while appendix S4 gives the necessary instructions to reproduce those analyses. 649 When dependent variables lasted until the occurrence of certain event, we 650 conducted survival analyses using the $\mathrm{R}$ package coxme (55). For the remaining 651 dependent variables, we did linear analyses using the R packages Ime4 (56) and 652 ImerTest (57). For the experiments done in homogeneous environment, we tested 653 the four different treatments (control, nutritive and adverse) as fixed factors. For the 654 spot experiments, we tested the effect of the nature of the nearest spot (neutral or $655 \mathrm{NaCl} 200 \mathrm{mM}$ ) and that of the furthest spot (glucose $100 \mathrm{mM}$ or $200 \mathrm{mM}$ ), as well as 
656 the interaction between the two. We always considered the date of the experiment

657 as a random factor as all treatments were conducted over multiple days, with several

658 replicates per day for each treatment. For each statistical analysis, we performed a 659 nested model comparison using the R package MuMln (58) by ordering models 660 according to their Akaike criterion. We represented the selected model by plotting 661 estimators with the pairwise comparison (a posteriori Tuckey test) p-values using the 662 R package emmeans (58) in linear models, and the hazard ratio associated p-values 663 in cox models.

664

665 Supporting information

666

667 S1 Appendix. Supplementary information: Image analysis and statistical

668 results. Description of the methodology to extract indexes from image analysis and

669 results obtained from the statistical analysis that support our observations.

670 S2 Video. Time-lapse of one experiment replicate, original images. Video

671 showing the evolution of the slime mold cell over the 35 hours of the experiments,

672 original acquired photos.

673 S3 Video. Time-lapse of one experiment replicate, trinarized images. Time

674 lapse of the results of the image segmentation for the same experiment shown in S2 675 Video.

676 S4 Appendix. Statistic analysis script description. Step by step description of the

677 procedure followed to generate the results of the shown statistical analyses. 


\section{Acknowledgements}

681

682 This work was supported by the U.S National Science Foundation, under grant

683 CMMI\#1552368: "CAREER: Multiphysics Damage and Healing of Rocks for

684 Performance Enhancement of Geo-Storage Systems - A Bottom-Up Research and

685 Education Approach." A.D. and A.B. were supported by a grant from the Agence

686 Nationale de la Recherche (reference number: ANR-17-CE02-0019-01 -SMART-

687 CELL).

688

689

690

691

692

693

694

695

696

697

698

699

700

701

702

703

704 


\section{References}

706

707 1. Ball P, Borley NR. The self-made tapestry: pattern formation in nature. Vol.

708 198. Oxford University Press Oxford; 1999.

709 2. Rietkerk M, de Koppel J. Regular pattern formation in real ecosystems. Trends Ecol Evol. 2008;23(3):169-75.

711 3. Theraulaz G, Gautrais J, Camazine S, Deneubourg J-L. The formation of spatial patterns in social insects: from simple behaviours to complex structures. Philos Trans R Soc London Ser A Math Phys Eng Sci. 2003;361(1807):1263-82.

4. Teague BP, Guye P, Weiss R. Synthetic morphogenesis. Cold Spring Harb Perspect Biol. 2016;8(9):a023929.

717 5. Goodwin BC. Unicellular morphogenesis. Cell Shape Determ Regul Regul Role. 1989;365-91.

719 6. Chalut KJ, Paluch EK. The actin cortex: a bridge between cell shape and function. Dev Cell. 2016;38(6):571-3.

721 7. Driscoll MK, McCann C, Kopace R, Homan T, Fourkas JT, Parent C, et al. Cell shape dynamics: from waves to migration. PLoS Comput Biol. 2012;8(3):e1002392.

724 8. Salbreux G, Charras G, Paluch E. Actin cortex mechanics and cellular morphogenesis. Trends Cell Biol. 2012;22(10):536-45.

726 9. Charras G, Sahai E. Physical influences of the extracellular environment on cell migration. Nat Rev Mol cell Biol. 2014;15(12):813.

728 10. van Helvert S, Storm C, Friedl P. Mechanoreciprocity in cell migration. Nat Cell Biol. 2018;20(1):8. 
730 11. Friedl P, Wolf K. Plasticity of cell migration: a multiscale tuning model. J Cell Biol. 2010;188(1):11-9.

12. Gardel ML, Schneider IC, Aratyn-Schaus Y, Waterman CM. Mechanical integration of actin and adhesion dynamics in cell migration. Annu Rev Cell Dev Biol. 2010;26:315-33.

13. Inagaki $\mathrm{N}$, Katsuno $\mathrm{H}$. Actin waves: Origin of cell polarization and migration? Trends Cell Biol. 2017;27(7):515-26.

14. Aman A, Piotrowski T. Cell migration during morphogenesis. Dev Biol. 2010;341(1):20-33.

15. Reig G, Pulgar E, Concha ML. Cell migration: from tissue culture to embryos. Development. 2014;141(10):1999-2013.

16. Yamada KM, Mayor R. Cell dynamics in development, tissue remodelling, and cancer. Curr Opin Cell Biol. 2016;42:iv.

17. Hind LE, Vincent WJB, Huttenlocher A. Leading from the back: the role of the uropod in neutrophil polarization and migration. Dev Cell. 2016;38(2):161-9.

18. Mseka T, Bamburg JR, Cramer LP. ADF/cofilin family proteins control formation of oriented actin-filament bundles in the cell body to trigger fibroblast polarization. J Cell Sci. 2007;120(24):4332-44.

19. King JS, Insall RH. Chemotaxis: finding the way forward with Dictyostelium. Trends Cell Biol. 2009;19(10):523-30.

$75020 . \quad$ Iglesias PA, Devreotes PN. Navigating through models of chemotaxis. Curr Opin Cell Biol. 2008;20(1):35-40.

752 21. Larsen SH, Adler J, Gargus JJ, Hogg RW. Chemomechanical coupling without 
755

756

757

758

759

760

761

762

763

764

765

766

767

768

769

770

771

772

773

774

775

776

777

778

779

22. Nakaoka Y, Iwatsuki K. Hyperpolarization-activated inward current associated with the frequency increase in ciliary beating of Paramecium. J Comp Physiol A. $1992 ; 170(6): 723-7$.

23. Saranak J, Foster KW. Rhodopsin guides fungal phototaxis. Nature. $1997 ; 387(6632): 465$.

24. Nakagaki T, Yamada H, Tóth A. Maze-solving by an amoeboid organism. Nature [Internet]. 2000;407(6803):470. Available from: http://www.ncbi.nlm.nih.gov/pubmed/11028990

25. Kuroda S, Takagi S, Nakagaki T, Ueda T. Allometry in Physarum plasmodium during free locomotion: size versus shape, speed and rhythm. J Exp Biol. 2015;218(23):3729-38.

26. Rodiek B, Hauser MJB. Migratory behaviour of Physarum polycephalum microplasmodia. Eur Phys J Spec Top. 2015;224(7):1199-214.

27. Vogel D, Nicolis SC, Perez-Escudero A, Nanjundiah V, Sumpter DJT, Dussutour A. Phenotypic variability in unicellular organisms: from calcium signalling to social behaviour. Proc R Soc B Biol Sci. 2015;282(1819):20152322.

28. Vogel D, Dussutour A, Deneubourg J-L. Symmetry breaking and inter-clonal behavioural variability in a slime mould. Biol Lett. 2018;14(12):20180504.

29. Aldrich H. Cell biology of Physarum and Didymium V1: organisms, nucleus, and cell cycle. Elsevier; 2012.

30. Oettmeier C, Brix K, Döbereiner H-G. Physarum polycephalum-A new take on a classic model system. J Phys D Appl Phys. 2017;50(41):413001.

31. Ueda T. Pattern dynamics in cellular perception. Phase Transitions A Multinatl J. 1993;45(2-3):93-104. 
780

781

782

783

784

785

786

787

788

789

790

791

792

793

794

795

796

797

798

799

800

801

802

803

804

32. Kuroda R, Kuroda H. Relation of cytoplasmic calcium to contractility in Physarum polycephalum. J Cell Sci. 1982;53(1):37-48.

33. Yoshimoto Y, Matsumura F, Kamiya N. Simultaneous oscillations of Ca2+ efflux and tension generation in the permealized plasmodial strand of Physarum. Cell Motil. 1981;1(4):433-43.

34. Farr DR, Amster H, Horisberger M. Composition and partial structure of the extracellular polysaccharide of Physarum polycephalum. Carbohydr Res. 1972;24(1):207-9.

35. Sesaki H, Ogihara S. Secretion of slime, the extracellular matrix of the plasmodium, as visualized with a fluorescent probe and its correlation with locomotion on the substratum. Cell Struct Funct. 1997;22(2):279-89.

36. Reid CR, Latty T, Dussutour A, Beekman M. Slime mold uses an externalized spatial "memory" to navigate in complex environments. Proc Natl Acad Sci. 2012;109(43):17490-4.

37. Reid CR, Beekman M. Solving the Towers of Hanoi - how an amoeboid organism efficiently constructs transport networks. J Exp Biol [Internet]. 2013;216:1546-51. Available from:

http://www.ncbi.nlm.nih.gov/pubmed/23307798

38. Ueda T, Hirose T, Kobatake Y. Membrane biophysicsdf chemoreception and taxis in the plasmodium of physarum polycephalum. Biophys Chem. 1980;11(3-4):461-73.

39. Reid CR, Latty T. Collective behaviour and swarm intelligence in slime moulds. FEMS Microbiol Rev. 2016;40(6):798-806.

40. Saigusa T, Tero A, Nakagaki T, Kuramoto Y. Amoebae anticipate periodic events. Phys Rev Lett. 2008;100(1):18101. 
805 41. Tero A, Takagi S, Saigusa T, Ito K, Bebber DP, Fricker MD, et al. Rules for biologically inspired adaptive network design. Science [Internet]. 2010 Jan 22 [cited 2018 Nov 21];327(5964):439-42. Available from: http://www.ncbi.nlm.nih.gov/pubmed/20093467

42. Latty T, Beekman M, Biernaskie J, Walker S, Gegear R, Boivin G, et al. Slime moulds use heuristics based on within-patch experience to decide when to leave. J Exp Biol [Internet]. 2015;218(Pt 8):1175-9. Available from: http://www.ncbi.nlm.nih.gov/pubmed/25722006

43. Reid CR, Garnier S, Beekman M, Latty T. Information integration and multiattribute decision making in non-neuronal organisms. Anim Behav. $2015 ; 100: 44-50$.

44. Boisseau RP, Vogel D, Dussutour A. Habituation in non-neural organisms: evidence from slime moulds. Proc R Soc B Biol Sci. 2016;283(1829):20160446.

45. Dussutour A, Ma Q, Sumpter D. Phenotypic variability predicts decision accuracy in unicellular organisms. Proc R Soc B. 2019;286(1896):20182825.

821 46. Terayama K, Ueda T, Kurihara K, Kobatake Y. Effect of sugars on salt reception in true slime moldPhysarum polycephalum. J Membr Biol. $1977 ; 34(1): 369-81$.

824 47. Carlile MJ. Nutrition and chemotaxis in the myxomycete Physarum polycephalum: the effect of carbohydrates on the plasmodium. Microbiology. 1970;63(2):221-6.

827 48. Ueda T, Terayama K, Kurihara K, Kobatake Y. Threshold phenomena in 828 chemoreception and taxis in slime mold Physarum polycephalum. J Gen $829 \quad$ Physiol. 1975;65(2):223-34. 
830 49. Ueda K, Takagi S, Nishiura Y, Nakagaki T. Mathematical model for contemplative amoeboid locomotion. Phys Rev E. 2011;83(2):21916.

832 50. Stephenson SL, Schnittler M. Myxomycetes. Handb Protists. 2017;1405-31.

833 51. Vogel D, Dussutour A. Direct transfer of learned behaviour via cell fusion in 834 non-neural organisms. Proc R Soc B Biol Sci. 2016;283(1845):20162382.

835 52. Kanungo T, Mount DM, Netanyahu NS, Piatko CD, Silverman R, Wu AY. An efficient k-means clustering algorithm: Analysis and implementation. IEEE Trans Pattern Anal Mach Intell. 2002;(7):881-92.

53. Reid CR, Beekman M, Latty T, Dussutour A. Amoeboid organism uses extracellular secretions to make smart foraging decisions. Behav Ecol. $2013 ; 24(4): 812-8$.

54. Dussutour A, Latty T, Beekman M, Simpson SJ. Amoeboid organism solves complex nutritional challenges. Proc Natl Acad Sci. 2010;107(10):4607-11.

843 55. Therneau T. A Package for Survival Analysis in S. version 2.38. 2015.

844 56. Bates D, Sarkar D, Bates MD, Matrix L. The Ime4 package. R Packag version. $2007 ; 2(1): 74$

846 57. Kuznetsova A, Brockhoff PB, Christensen RHB. ImerTest package: tests in linear mixed effects models. J Stat Softw. 2017;82(13).

848 58. Barton K, Barton MK. Package 'MuMIn'Version 1. 2015;

849 59. Halvorsrud R, Wagner G. Growth patterns of the slime mold Physarum on a 850 nonuniform substrate. Phys Rev E. 1998;57(1):941.

851 60. Nakagaki T, Yamada H, Ueda T. Interaction between cell shape and 852 contraction pattern in the Physarum plasmodium. Biophys Chem. 2000;84(3):195-204.

854 61. Latty T, Beekman M. Food quality affects search strategy in the acellular slime 
mould, Physarum polycephalum. Behav Ecol. 2009;20(6):1160-7.

62. Knowles DJC, Carlile MJ. The chemotactic response of plasmodia of the Microbiology. 1978;108(1):17-25.

63. Daniel JW, Baldwin HH. Methods of culture for plasmodial myxomycetes. In: Methods in Cell Biology. Elsevier; 1964. p. 9-41.

64. Takamatsu A, Takaba E, Takizawa G. Environment-dependent morphology in plasmodium of true slime mold Physarum polycephalum and a network growth model. J Theor Biol. 2009;256(1):29-44.

65. Nwodo U, Green E, Okoh A. Bacterial exopolysaccharides: functionality and prospects. Int J Mol Sci. 2012;13(11):14002-15.

66. McCormick JJ, Blomquist JC, Rusch HP. Isolation and characterization of a galactosamine wall from spores and spherules of Physarum polycephalum. J Bacteriol. 1970;104(3):1119-25.

67. Simon HL, Henney HR. Chemical composition of slime from three species of myxomycetes. FEBS Lett. 1970;7(1):80-2.

68. Asgari M, Henney JHR. Inhibition of growth and cell wall morphogenesis of Cytobios. 1977;20(79-80):163-77.

874 69. Haskins EF, Hinchee AA. Light-and ultra-microscopical observations on the surface structure of the protoplasmodium, aphanoplasmodium, and phaneroplasmodium (Myxomycètes). Can J Bot. 1974;52(8):1835-9. 